\title{
On the Stark effect in open shell complexes exhibiting partially quenched electronic angular momentum: Infrared laser Stark spectroscopy of $\mathrm{OH}-\mathrm{C}_{2} \mathrm{H}_{2}, \mathrm{OH}-\mathrm{C}_{2} \mathrm{H}_{4}$, and $\mathrm{OH}-\mathrm{H}_{2} \mathrm{O}$
}

Christopher P. Moradi and Gary E. Douberly a)

Department of Chemistry, University of Georgia, Athens, Georgia 30602-2556, USA

a) Email: douberly@uga.edu; Tele: 01-706-542-3857

\section{Abstract}

The Stark effect is considered for polyatomic open shell complexes that exhibit partially quenched electronic angular momentum. Matrix elements of the Stark Hamiltonian represented in a parity conserving Hund's case (a) basis are derived for the most general case, in which the permanent dipole moment has projections on all three inertial axes of the system. Transition intensities are derived, again for the most general case, in which the laser polarization has projections onto axes parallel and perpendicular to the Stark electric field, and the transition dipole moment vector is projected onto all three inertial axes in the molecular frame.

Simulations derived from this model are compared to experimental rovibrational Stark spectra of $\mathrm{OH}-\mathrm{C}_{2} \mathrm{H}_{2}, \mathrm{OH}-\mathrm{C}_{2} \mathrm{H}_{4}$, and $\mathrm{OH}-\mathrm{H}_{2} \mathrm{O}$ complexes formed in helium nanodroplets. 


\section{Introduction}

The Stark effect has been exploited to probe the dipole moments of a broad array of molecular systems using spectroscopic techniques that span the electromagnetic spectrum [1-3]. The inertial components of the dipole moment are the essential ingredients necessary for calculations of absolute intensities in rotational spectroscopy [1, 4]. Experimental dipole moments obtained from Stark spectroscopy also provide stringent benchmarks for electronic structure theory [5-7]. The quantum mechanical operator describing the dipole moment is a sum of one-electron operators, and its electronic expectation value converges rather quickly with increasing basis set size in a variational ab initio calculation $[8,9]$. Moreover, for systems that exhibit wide-amplitude motion, the vibrational expectation value of the dipole moment provides a sensitive probe of the ground state nuclear wavefunction $[10,11]$.

A number of techniques have recently emerged that exploit the Stark effect to manipulate molecular beams to produce and trap ensembles of cold neutral molecules (see Ref. [12] and references therein). Many of the systems of interest are open-shell radicals, as these are particularly intriguing as targets for cold collision studies [13-15]. For open-shell diatomic or covalently bonded polyatomic systems subjected to electric and magnetic fields, the theoretical framework necessary to describe the rotational energy levels is well established $[1,16]$, although this remains an active area of research [17]. The Stark effect for weakly bound open-shell complexes has also been discussed $[18,19]$, although not nearly as extensively as for the aforementioned strongly bound systems.

We investigate here the Stark effect for $C_{2 v}$ molecular complexes. These complexes are composed of a closed-shell polyatomic molecule and the hydroxyl radical, which is aligned 
along the inertial $a$-axis ( $C_{2}$ axis). Provided the intermolecular interaction in these systems is sufficiently weak, the orbital and spin angular momenta remain separately quantized along the $\mathrm{OH}$ bond axis. However, as the strength of the intermolecular interaction increases, the electronic angular momentum becomes partially quenched due to the incipient barrier to free orbital motion of the electron about the $\mathrm{OH}$ axis. A zero-field model Hamiltonian and simulated spectra for these systems have been reported by Marshall and Lester [20, 21], and their model has been applied successfully to interpret the rovibrational infrared (IR) spectra of the $T$-shaped $\mathrm{OH}-\mathrm{C}_{2} \mathrm{H}_{2}$ complex $[22,23]$ and the microwave spectra of the $\mathrm{OH}-\mathrm{H}_{2} \mathrm{O}$ complex [24-26]. Using a model Hamiltonian adapted from this previous zero-field work, simulations are shown to be in excellent agreement with the Stark spectra of the $\mathrm{OH}-\mathrm{C}_{2} \mathrm{H}_{2}, \mathrm{OH}-\mathrm{C}_{2} \mathrm{H}_{4}$, and $\mathrm{OH}-\mathrm{H}_{2} \mathrm{O}$ complexes formed in helium nanodroplets.

\section{Rotational Basis and Coordinate System}

The coordinate system employed here is the same as that described previously for the $T$ shaped $\mathrm{OH}-\mathrm{C}_{2} \mathrm{H}_{2}$ complex [20, 23], which is shown in Figure 1. All of the $\mathrm{OH}-\mathrm{X}$ systems considered here are assumed to have $C_{2 v}$ symmetry. Moreover, it is also assumed that these systems are semi-rigid, in the sense that any angular momentum coupling involving the internal rotational motion of the $\mathrm{OH}$ radical can be ignored.

The molecule-fixed $z$-axis is explicitly defined as the inertial $a$-axis ( $C_{2}$ axis), and the tensor operators for the permanent electric dipole moment vector in the inertial frame of reference are given in equations 1 and 2 . 
$\mathrm{T}_{0}^{1}(\boldsymbol{\mu})=\mu_{a}$

$\mathrm{T}_{ \pm 1}^{1}(\boldsymbol{\mu})=\mp \frac{1}{\sqrt{2}}\left(\mu_{b} \pm i \mu_{c}\right)$.

The laboratory $Z$-axis is defined as the direction of the homogeneous, dc Stark field. The tensor operators for the laboratory frame Stark field vector are given in equations 3 and 4.

$\mathrm{T}_{0}^{1}(\boldsymbol{E})=E_{Z}=E$, and

$\mathrm{T}_{ \pm 1}^{1}(\boldsymbol{E})=\mp \frac{1}{\sqrt{2}}\left(E_{X} \pm i E_{Y}\right)=0$.

In the limit of weak intermolecular interaction, projections of spin and orbital angular momenta onto the $\mathrm{OH}$ bond axis remain separately quantized. Therefore, Hund's case (a) primitive basis functions are employed, which take the form of the following product,

$|J p M \lambda \sigma\rangle=|J p M\rangle|\eta \lambda\rangle|s \sigma\rangle$.

The $J, p$, and $M$ quantum numbers are all half-integral and correspond to the total angular momentum (less nuclear spin) and its projections on the inertial frame $a$-axis and the laboratory frame $Z$-axis, respectively. The symmetric rotor part of the primitive functions is given by,

$|J p M\rangle=\left(\frac{2 J+1}{8 \pi^{2}}\right)^{1 / 2} \mathcal{D}_{M, p}^{(J) *}(\alpha)$,

where, $\alpha$ represents the Euler angles. The projections of orbital, $l=1$, and spin, $s=1 / 2$, angular momenta onto the inertial $a$-axis are given by the quantum numbers $\lambda= \pm 1$ and $\sigma=$ $\pm 1 / 2$, respectively. It is also convenient to define the quantum number $\omega=\lambda+\sigma$. The quantum number $\eta$ allows for a more general treatment of the problem, in which multiple electronic states having the same value of $\lambda$ are included [18]. 
The Hamiltonian matrix is represented in a parity conserving basis that consists of linear combinations of the above primitive functions (eqn. 7, with $P=|p| \geq 1 / 2$ and $\epsilon= \pm 1$ ).

$|J P M \lambda \sigma \epsilon\rangle=\frac{1}{\sqrt{2}}\left\{|J P M \lambda \sigma\rangle+\epsilon(-1)^{(J-1 / 2)}|J,-P, M,-\lambda,-\sigma\rangle\right\}$

With $\lambda= \pm 1$, the rotationless parity, $\chi\left(E^{*}\right)$, of the basis function is $\epsilon(-1)^{P+\sigma}$. These parity conserving functions transform as irreducible representations in $C_{2 v}$. Following Ref. [20], the basis set transformation properties are given in Table I. Again, this parity conserving, Hund's case (a) representation is appropriate for the zero-field, weak intermolecular interaction limit, in which orbital motion of the electron about the $\mathrm{OH}$ bond axis is nearly isotropic.

In the following treatment of the Stark effect, we neglect the sequential coupling of nuclear spin angular momenta to $J$. For the hydroxyl radical containing complexes discussed here, we estimate that the zero-field hyperfine splitting due to this neglected coupling is $\approx 10^{2}$ to $10^{3}$ times less than the experimental spectral line widths in helium droplets, which are limited by inhomogeneous broadening effects. A full treatment of the problem in the presence of this coupling requires the derivation of matrix elements in a parity conserving basis composed of the primitive functions, $\left|(J I) F p M_{F} \lambda \sigma\right\rangle=\left|(J I) F p M_{F}\right\rangle|\eta \lambda\rangle|s \sigma\rangle$, where $F=J+I$, and $I$ is the nuclear spin angular momentum. Matrix elements of the Stark Hamiltonian in this primitive coupled basis can be found in the textbook by Brown and Carrington [1], and matrix elements in a parity conserving basis, composed of these primitive functions, can be derived in a similar way to the procedure described here. We note, however, that for rather modest field strengths $(\approx 1 \mathrm{kV} / \mathrm{cm})$, it is reasonable to expect the nuclear spin angular momentum to be decoupled from the rotational angular momentum, and the uncoupled representation used here will be rigorously appropriate in the high-field limit [3]. 


\section{Energies and Eigenvectors}

The model Hamiltonian employed here (eqn. 8) contains the Stark Hamiltonian, $\widehat{H}_{\text {Stark }}$, and terms associated with the zero-field problem, namely those that account for asymmetric top rigid rotation, centrifugal distortion, spin-orbit coupling, and electronic angular momentum quenching $\left(\widehat{H}_{\text {rot }}, \widehat{H}_{\mathrm{CD}}, \widehat{H}_{\mathrm{SO}}\right.$, and $\widehat{H}_{q}$, respectively).

$\widehat{H}=\widehat{H}_{\text {rot }}+\widehat{H}_{\mathrm{CD}}+\widehat{H}_{\mathrm{SO}}+\widehat{H}_{q}+\widehat{H}_{\text {Stark }}$

The procedure by which eigenvalues and eigenvectors of $\boldsymbol{H}$ are obtained starts by transforming the $\boldsymbol{H}_{0}=\boldsymbol{H}-\boldsymbol{H}_{\text {Stark }}$ matrix to the zero-field eigenvector basis, $\boldsymbol{A}^{\dagger} \boldsymbol{H}_{0} \boldsymbol{A}=\boldsymbol{H}^{\prime}{ }_{0}$. The Stark Hamiltonian matrix is then transformed to this zero-field eigenvector basis, $\boldsymbol{A}^{\dagger} \boldsymbol{H}_{\text {Stark }} \boldsymbol{A}=$ $\boldsymbol{H}_{\text {Stark }}$, added to the diagonalized zero-field matrix, and then transformed to the Stark eigenvector basis, $\boldsymbol{B}^{\dagger}\left(\boldsymbol{H}^{\prime}{ }_{\text {Stark }}+\boldsymbol{H}^{\prime}{ }_{0}\right) \boldsymbol{B}=\boldsymbol{H}^{\prime \prime}{ }_{\text {Stark }}$. Eigenvalue and eigenvector matrices are found for both the ground $\left(\boldsymbol{A}_{g}, \boldsymbol{B}_{g}\right)$ and excited $\left(\boldsymbol{A}_{\boldsymbol{e}}, \boldsymbol{B}_{\boldsymbol{e}}\right)$ vibrational states, where the set of rotational constants and dipole moments are allowed to differ.

\section{IIIa. Zero-Field Hamiltonian}

The terms associated with the zero-field problem have been discussed in detail previously $[20,21,23,25]$. Only the lowest energy, doubly degenerate $\eta=1$ state is considered, and only the diagonal part of the spin-orbit Hamiltonian is retained, leading to an effective two electronic state model. The off-diagonal spin-orbit coupling terms that connect states differing in $|\lambda|$ are ignored. 
The spin-orbit Hamiltonian and its matrix elements are given in eqns. 9 and 10, respectively.

$$
\begin{aligned}
& \widehat{H}_{\mathrm{SO}}=A_{\mathrm{SO}} \mathrm{T}_{0}^{1}(\boldsymbol{L}) \mathrm{T}_{0}^{1}(\boldsymbol{S}) \\
& \left\langle J P M \lambda \sigma \epsilon\left|\widehat{H}_{\mathrm{SO}}\right| J^{\prime} P^{\prime} M^{\prime} \lambda^{\prime} \sigma^{\prime} \epsilon^{\prime}\right\rangle=A_{\mathrm{SO}} \lambda \sigma \delta_{J, J^{\prime}} \delta_{P, P^{\prime}} \delta_{M, M^{\prime}} \delta_{\lambda, \lambda^{\prime}} \delta_{\sigma, \sigma^{\prime}} \delta_{\epsilon, \epsilon^{\prime}}
\end{aligned}
$$

As usual, the spin-orbit term lifts the two-fold $|\omega|=|\lambda+\sigma|$ degeneracy associated with the $\mathrm{OH}$ electronic angular momentum.

The spherical tensor form of the quenching operator and its matrix elements when $l=1$ are given in eqns. 11 and 12, respectively.

$$
\begin{aligned}
& \widehat{H}_{q}=\frac{\rho}{l(l+1)}\left[\mathrm{T}_{2}^{2}(\boldsymbol{L})+\mathrm{T}_{-2}^{2}(\boldsymbol{L})\right] \\
& \left\langle J P M \lambda \sigma \epsilon\left|\widehat{H}_{q}\right| J^{\prime} P^{\prime} M^{\prime} \lambda^{\prime} \sigma^{\prime} \epsilon^{\prime}\right\rangle=\frac{\rho}{2} \delta_{J, J^{\prime}} \delta_{P, P^{\prime}} \delta_{M, M^{\prime}} \delta_{\lambda,-\lambda^{\prime}} \delta_{\sigma, \sigma^{\prime}} \delta_{\epsilon, \epsilon^{\prime}}
\end{aligned}
$$

This angular momentum quenching term accounts for the anisotropy for orbital motion of the unpaired electron about the inertial $a$-axis. This anisotropy quenches the orbital angular momentum as the hydroxyl radical binds to either $\mathrm{C}_{2} \mathrm{H}_{2}, \mathrm{C}_{2} \mathrm{H}_{4}$ or $\mathrm{H}_{2} \mathrm{O}$ in a $C_{2 v}$ configuration. The extent of this quenching can be modeled via the magnitude of $\rho$, which is often referred to as the “difference potential" [20]. In the weak interaction limit, the spectroscopic fitting parameter $\rho$ has a magnitude close to zero, and the two electronic states in the model are approximately separated by the spin-orbit coupling constant, $A_{\mathrm{SO}}$, of the isolated $\mathrm{OH}$ radical (i.e. in the absence of rotation). As the interaction energy increases, $\rho$ increases in magnitude, and the energetic separation between electronic states becomes $\approx \sqrt{A_{S O}^{2}+\rho^{2}}$ [20]. In the strong-interaction limit, when $\rho \gg A_{\text {So, }}$ orbital angular momentum about the $\mathrm{OH}$ bond axis is fully quenched, and the 
energetic difference between ${ }^{2} B_{2}$ and ${ }^{2} B_{1}$ electronic states is $\approx \rho$. These states in the fully quenched limit can be thought of as consisting of a singly occupied $p \pi$ orbital centered on the $\mathrm{OH}$ moiety, which is aligned either in or out of the molecular plane, respectively. Moreover, in this limit, the spin angular momentum is completely decoupled from the molecular framework and instead is coupled to the rotational angular momentum of the complex (i.e. Hund's case (b) coupling). The parameter $\rho$ in the quenching Hamiltonian therefore allows for a smooth transition in the model between the weak (Hund's case (a) coupling) and strong (Hund's case (b) coupling) interaction limits. For all three systems considered here, the two lowest energy electronic states $\left({ }^{2} B_{1}\right.$ and $\left.{ }^{2} B_{2}\right)$ at equilibrium correlate, respectively, to the ${ }^{2} \Pi_{3 / 2}$ and ${ }^{2} \Pi_{1 / 2}$ levels of the $\mathrm{OH}$ fragment as the intermolecular separation increases [23].

The matrix elements of $\widehat{H}_{\text {rot }}$ and $\widehat{H}_{\mathrm{CD}}$ used here are the same as those discussed previously $[20,23]$. The rotational and centrifugal distortion constants determined from simulations of the zero-field spectra are retained in simulations of Stark spectra without modification.

There are two effects worth noting for the rotating molecule. The first is a Coriolis interaction of the form $-2 A \sum_{p=-1}^{1}(-1)^{p} \mathrm{~T}_{p}^{1}(J) \mathcal{D}_{-p 0}^{(1) *}\left(\mathrm{~T}_{0}^{1}(\boldsymbol{L})+\mathrm{T}_{0}^{1}(\boldsymbol{S})\right)$, which lifts the $\pm \omega$ degeneracy [20,23]. For example, the $\left(P, \omega= \pm \frac{3}{2}\right)$ manifolds are split by $\approx 3 A$ and $\approx 9 A$ for $P=\frac{1}{2}$ and $P=\frac{3}{2}$, respectively. This Coriolis effect also dramatically reduces the role of matrix elements proportional to the inertial asymmetry that connect states differing by two in the $P$ quantum number (eqn. 13).

$\left\langle J P M \lambda \sigma \epsilon\left|\widehat{H}_{\text {rot }}\right| J^{\prime} P^{\prime} M^{\prime} \lambda^{\prime} \sigma^{\prime} \epsilon^{\prime}\right\rangle=$

$\left(\frac{B-C}{4}\right)[J(J+1)-P(P \pm 1)]^{1 / 2}[J(J+1)-(P \pm 1)(P \pm 2)]^{1 / 2} \delta_{J, J^{\prime}} \delta_{P \pm 2, P^{\prime}} \delta_{M, M^{\prime}} \delta_{\lambda, \lambda^{\prime}} \delta_{\sigma, \sigma^{\prime}} \delta_{\epsilon, \epsilon^{\prime}}$ 
These matrix elements are analogous to those in the closed-shell asymmetric top Hamiltonian that lead to asymmetry splitting. As a result of the Coriolis contribution to the energy, these asymmetry matrix elements (on the order of $10^{-4}$ to $10^{-3} \mathrm{~cm}^{-1}$ ) connect zero-order states differing in energy by several units of the $A$ rotational constant (which is $\approx 1 \mathrm{~cm}^{-1}$ for $\mathrm{OH}-\mathrm{C}_{2} \mathrm{H}_{2}$ ). Therefore, the asymmetry splitting in these open-shell systems is effectively eliminated.

In the rotating molecule, a non-zero value for $\rho$ leads to substantial parity splitting of levels belonging to the $\left(P=\frac{1}{2}, \omega=+\frac{3}{2},-\frac{1}{2}\right)$ manifolds [20,23] vide infra. This parity splitting is fully resolved in the zero-field spectra of He-solvated $\mathrm{OH}-\mathrm{C}_{2} \mathrm{H}_{2}$ [23]. Combination differences from the $a$-type $\mathrm{OH}$ stretch band revealed the vibrationally averaged value of $\rho$ to be $-165 \mathrm{~cm}^{-1}$ for the $\mathrm{OH}-\mathrm{C}_{2} \mathrm{H}_{2}$ complex. Following the established convention [27], the negative sign is indicative of the ${ }^{2} B_{1}$ electronic state being lower in energy than ${ }^{2} B_{2}$. This zero-field value for $\rho$ is retained in the Stark simulations reported here. Moreover, as justified previously [23], the spin-orbit coupling constant, $A_{\mathrm{s} 0}$, is fixed to the $\mathrm{OH}$ monomer value, $-139.05 \mathrm{~cm}^{-1}$. Figure 2 shows zero-field simulations of the $\mathrm{OH}-\mathrm{C}_{2} \mathrm{H}_{2}, a$-type spectra for various values of the difference potential. As discussed in detail previously [20,23], for $\rho=0$, the spectrum resembles a symmetric top in a degenerate vibronic state characterized by half-integer quantum numbers $J, P$ and $\omega$. With increasing $\rho$, the spectrum evolves into that expected for an asymmetric top characterized by the integer quantum numbers $N$ and $K_{a}$. 


\section{IIIb. Stark Hamiltonian}

We express the Stark Hamiltonian (eqn. 14) in spherical tensor operator notation, where $p$ and $q$ are indices for the lab and inertial frames of reference, respectively.

$\widehat{H}_{\text {Stark }}=-\mathrm{T}^{1}(\boldsymbol{\mu}) \cdot \mathrm{T}^{1}(\boldsymbol{E})=-\sum_{p=-1}^{1}(-1)^{p} \mathrm{~T}_{-p}^{1}(\boldsymbol{E}) \sum_{q=-1}^{1} \mathcal{D}_{p q}^{(1) *} \mathrm{~T}_{q}^{1}(\boldsymbol{\mu})$

Expansion of eqn. 14 gives the Stark operator associated with the most general case, in which the dipole moment has projections on all three inertial axes.

$\widehat{H}_{\text {Stark }}=-\sum_{p=-1}^{1}(-1)^{p} \mathrm{~T}_{-p}^{1}(\boldsymbol{E})\left[\mathcal{D}_{p 0}^{(1) *} \mu_{a}+\frac{1}{\sqrt{2}}\left(\mathcal{D}_{p-1}^{(1) *}-\mathcal{D}_{p+1}^{(1) *}\right) \mu_{b}-\frac{i}{\sqrt{2}}\left(\mathcal{D}_{p-1}^{(1) *}+\mathcal{D}_{p+1}^{(1) *}\right) \mu_{c}\right]$

Matrix elements in the primitive basis (eqn. 5) can be derived for individual $\mathcal{D}_{p q}^{(1) *}$ terms by applying the Wigner-Eckart theorem [1]. After considerable algebra, elements of the Wigner rotation matrices in the parity conserving basis (eqn. 7) are obtained, where

$\left\langle J P M \lambda \sigma \epsilon\left|\mathcal{D}_{p 0}^{(1) *}\right| J^{\prime} P^{\prime} M^{\prime} \lambda^{\prime} \sigma^{\prime} \epsilon^{\prime}\right\rangle=(-1)^{M-P}\left[(2 J+1)\left(2 J^{\prime}+1\right)\right]^{1 / 2}\left(\begin{array}{ccc}J & 1 & J^{\prime} \\ -M & p & M^{\prime}\end{array}\right)\left(\begin{array}{ccc}J & 1 & J^{\prime} \\ -P & 0 & P^{\prime}\end{array}\right) \delta_{\lambda, \lambda^{\prime}} \delta_{\sigma, \sigma^{\prime}} \delta_{\epsilon,-\epsilon^{\prime}}$

and,

$\left\langle J P M \lambda \sigma \epsilon\left|\left(\mathcal{D}_{p-1}^{(1) *} \pm \mathcal{D}_{p+1}^{(1) *}\right)\right| J^{\prime} P^{\prime} M^{\prime} \lambda^{\prime} \sigma^{\prime} \epsilon^{\prime}\right\rangle=(-1)^{M-P}\left[(2 J+1)\left(2 J^{\prime}+1\right)\right]^{1 / 2}\left(\begin{array}{ccc}J & 1 & J^{\prime} \\ -M & p & M^{\prime}\end{array}\right)\left(\frac{1 \mp \epsilon \epsilon^{\prime}}{2}\right)\left\{\left[\left(\begin{array}{ccc}J & 1 & J^{\prime} \\ -P & -1 & P^{\prime}\end{array}\right) \pm\right.\right.$

$\left.\left.\left(\begin{array}{ccc}J & 1 & J^{\prime} \\ -P & 1 & P^{\prime}\end{array}\right)\right] \delta_{\lambda, \lambda^{\prime}} \delta_{\sigma, \sigma^{\prime}}-\epsilon(-1)^{J-1 / 2}\left(\begin{array}{ccc}J & 1 & J^{\prime} \\ P & -1 & P^{\prime}\end{array}\right) \delta_{\lambda,-\lambda^{\prime}} \delta_{\sigma,-\sigma^{\prime}}\right\}$.

Equations 16 and 17 are used to obtain matrix elements of $\boldsymbol{H}_{\text {Stark }}$ in the parity conserving basis. Again, the model discussed here is compared to experimental spectra of $\mathrm{OH}-\mathrm{C}_{2} \mathrm{H}_{2}, \mathrm{OH}-\mathrm{C}_{2} \mathrm{H}_{4}$, and $\mathrm{OH}-\mathrm{H}_{2} \mathrm{O}$ complexes in $\mathrm{He}$ nanodroplets. These complexes all have permanent dipole moments that lie along the inertial $a$-axis $(q=0)$. With the Stark field direction defined as the Zaxis in the lab frame $(p=0)$, the following $\boldsymbol{H}_{\text {Stark }}$ matrix elements are obtained. 
$\left\langle J P M \lambda \sigma \epsilon\left|\widehat{H}_{S t a r k}\right| J^{\prime} P^{\prime} M^{\prime} \lambda^{\prime} \sigma^{\prime} \epsilon^{\prime}\right\rangle=$

$-\mu_{a} E(-1)^{M-P}\left[(2 J+1)\left(2 J^{\prime}+1\right)\right]^{1 / 2}\left(\begin{array}{ccc}J & 1 & J^{\prime} \\ -M & 0 & M^{\prime}\end{array}\right)\left(\begin{array}{ccc}J & 1 & J^{\prime} \\ -P & 0 & P^{\prime}\end{array}\right) \delta_{\lambda, \lambda^{\prime}} \delta_{\sigma, \sigma^{\prime}} \delta_{\epsilon,-\epsilon^{\prime}}$

The zero-field Hamiltonian matrix, $\boldsymbol{H}_{0}$, represented in the parity conserving basis (eqn. 7), is diagonal in $J, M,(-1)^{P+\sigma}$, and $\epsilon$. In the presence of the field, however, $\boldsymbol{H}$ is diagonal only in $M$, which remains a good quantum number under non-zero-field conditions. The application of the Stark field mixes zero-field states differing in $J$ by 0 or 1; moreover, states differing in $\epsilon$ are mixed by the Stark effect, although the value of $(-1)^{P+\sigma}$ is conserved. Therefore, the Stark effect, as expected, mixes zero-field states of opposite parity. It is instructive to consider a $4 \times 4$ block of $\boldsymbol{H}$ in order to fully appreciate the Stark effect in a system exhibiting partially quenched electronic angular momentum. The matrix elements in eqn. 19 correspond to the quantum numbers $|J, P, M\rangle=\left|\frac{1}{2}, \frac{1}{2}, \pm \frac{1}{2}\right\rangle$. As discussed previously, $\boldsymbol{J} \cdot \boldsymbol{S}$ terms in $\widehat{H}_{\text {rot }}$ lead to the paritydependent off-diagonal matrix elements proportional to $(B+C) / 2$, which cause parity splitting of rotational levels on the order of $0.001 \mathrm{~cm}^{-1}$ in the $\rho=0$ limit $[20,23]$. However, in the presence of a non-zero difference potential (say $|\rho|=120 \mathrm{~cm}^{-1}$ ), these matrix elements, $\langle\omega=+3 / 2, \epsilon= \pm 1|H| \omega=-1 / 2, \epsilon= \pm 1\rangle=\rho / 2 \mp(B+C) / 2$, cause parity splitting on the order of $0.1 \mathrm{~cm}^{-1}$.

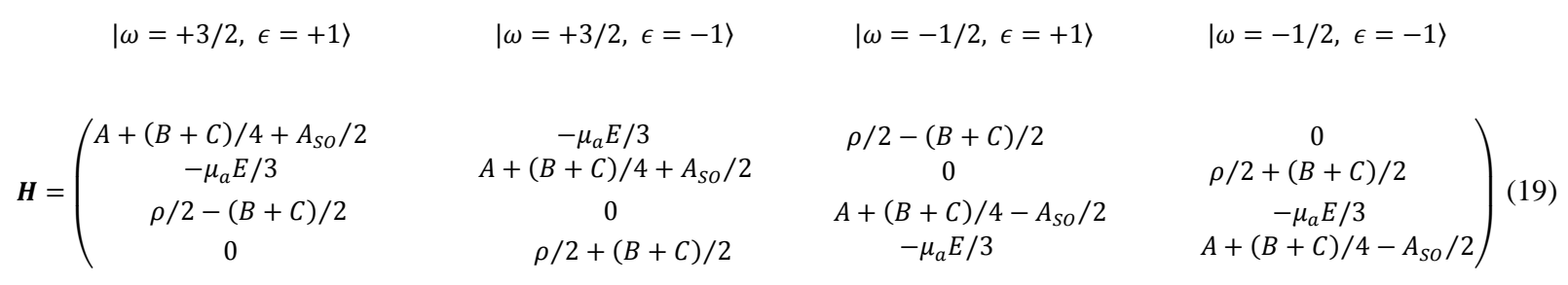


Due to this rather large parity splitting, already present under zero-field conditions, the Stark effect is significantly diminished for these levels, because the mixing of states of opposite parity is less than it would be in the absence of angular momentum quenching (i.e. when $\rho=0$ ). It is interesting to note that all other parity-dependent off-diagonal matrix elements in $\boldsymbol{H}$ are proportional to the inertial asymmetry, $(B-C)$, which is small for the near-prolate tops considered here. For example, parity splitting in the $\left(P=\frac{3}{2}, \omega=+\frac{3}{2}\right)$ manifold of levels is too small to be observed in the $\mathrm{OH}-\mathrm{C}_{2} \mathrm{H}_{2}$ spectra. Indeed, the model predicts this splitting to be less than $100 \mathrm{kHz}$. As a result, the Stark splitting of these levels, for a given field strength, is expected to be significantly larger than for the parity doubled levels in the $\left(P=\frac{1}{2}, \omega=+\frac{3}{2}\right)$ manifold, due to the much smaller energy difference between nearby states of opposite parity. This can be seen in Figure 3, which shows the Stark splitting of the $\left|J=\frac{1}{2}, P=\frac{1}{2}, \omega=\frac{3}{2}\right\rangle$ and $\left|J=\frac{3}{2}, P=\frac{3}{2}, \omega=\frac{3}{2}\right\rangle$ levels, which are the lowest energy levels of $A$ and $B$ nuclear spin symmetry, respectively. Indeed, the splitting of the lower energy $\left|J=\frac{3}{2}, P=\frac{3}{2}, \omega=\frac{3}{2}\right\rangle$ level appears similar to the linear, first-order Stark effect associated with the degenerate $|K| \neq 0$ levels of a closed-shell symmetric top, whereas the Stark splitting of the parity doubled $\left|P=\frac{1}{2}, \omega=\frac{3}{2}\right\rangle$ levels resembles a quadratic Stark effect.

\section{Stark Spectra Transition Intensities}

Rovibrational transition intensities are obtained via the matrix, $\boldsymbol{M}$, which is represented in the parity conserving basis. The $\boldsymbol{M}$ matrix is transformed via the matrix operation, 
$\boldsymbol{B}_{\boldsymbol{e}}^{\dagger} \boldsymbol{A}_{\boldsymbol{e}}^{\dagger} \boldsymbol{M} \boldsymbol{A}_{\boldsymbol{g}} \boldsymbol{B}_{\boldsymbol{g}}=\boldsymbol{I}$. The elements of the intensity matrix, $\boldsymbol{I}$, correspond to $\sqrt{\frac{I_{i j}}{g w}}$, where $I_{i j}$ is the intensity of the transition from the $j^{\text {th }}$ ground-state level to the $i^{\text {th }}$ excited-state level, and $g$ and $w$ are nuclear spin statistical and Boltzmann weights, respectively. The Stark effect reduces the symmetry of the physical system to $C_{2}$, and rovibronic states transforming as $A_{1}$ and $A_{2}$ under zero-field conditions correlate to states of $A$ symmetry under non-zero-field conditions. Similarly, $B_{1}$ and $B_{2}$ rovibronic states correlate to states of $B$ symmetry. Although somewhat obvious that this must be the case, this symmetry analysis confirms that the Stark effect cannot mix states belonging to different nuclear spin isomers, and nuclear spin statistical weights assigned to states in the zero-field limit (on the basis of the sign of $(-1)^{P+\sigma}$ ) can be carried through to the calculation of intensities in the Stark spectra. The spin statistical weight ratio for the $\mathrm{OH}-\mathrm{C}_{2} \mathrm{H}_{2}$ and $\mathrm{OH}-\mathrm{H}_{2} \mathrm{O}$ complexes is 3:1 for odd:even $(P+\sigma)$ levels. For the $\mathrm{OH}-\mathrm{C}_{2} \mathrm{H}_{4}$ complex, the ratio is 10:6 for odd:even $(P+\sigma)$ levels. The Boltzmann weights are obtained separately for each nuclear spin modification with a rotational temperature equal to $0.35 \mathrm{~K}$, and it is assumed that rotational state populations are transferred adiabatically as the system enters into the Stark field region of the apparatus. On the basis of the agreement between experiment and simulation, this appears to be a good assumption.

The tensor form of the $\widehat{M}$ operator is,

$\widehat{M}=\mathrm{T}^{1}(\boldsymbol{\mu}) \cdot \mathrm{T}^{1}(\boldsymbol{E})=\sum_{p=-1}^{1}(-1)^{p} \mathrm{~T}_{-p}^{1}(\boldsymbol{E})\left[\mathcal{D}_{p 0}^{(1) *} \mu_{a}+\frac{1}{\sqrt{2}}\left(\mathcal{D}_{p-1}^{(1) *}-\mathcal{D}_{p+1}^{(1) *}\right) \mu_{b}-\frac{i}{\sqrt{2}}\left(\mathcal{D}_{p-1}^{(1) *}+\mathcal{D}_{p+1}^{(1) *}\right) \mu_{c}\right]$.

The operators $\mathrm{T}^{1}(\boldsymbol{\mu})$ and $\mathrm{T}^{1}(\boldsymbol{E})$ in eqn. 20 are associated with the vibrational transition dipole moment and the laser electric field, respectively. Therefore, $\mu_{a}, \mu_{b}$, and $\mu_{c}$ correspond here to the components of the dipole derivative vector associated with a particular vibrational excitation. Of course, for pure rotational spectroscopy, these are instead the vibrationally averaged inertial 
components of the permanent electric dipole moment. Equation 20 corresponds again to the most general case; namely, the laser polarization has projections on axes parallel $(p=0)$ and perpendicular $(p= \pm 1)$ to the Stark electric field, and the transition dipole moment vector has projections on the $a$-, $b$ - and $c$ - axes in the inertial frame. Noting the spherical tensor operator form of the electric field vector (eqns. 3 and 4), the matrix elements of $\boldsymbol{M}$ can be individually derived for the various band-types and laser polarization configurations (using eqns. 16 and 17):

i) $a$-type, parallel polarization:

$\mu_{a} E_{Z}(-1)^{M-P}\left[(2 J+1)\left(2 J^{\prime}+1\right)\right]^{1 / 2}\left(\begin{array}{ccc}J & 1 & J^{\prime} \\ -M & 0 & M^{\prime}\end{array}\right)\left(\begin{array}{ccc}J & 1 & J^{\prime} \\ -P & 0 & P^{\prime}\end{array}\right) \delta_{\lambda, \lambda^{\prime}} \delta_{\sigma, \sigma^{\prime}} \delta_{\epsilon,-\epsilon^{\prime}}$

ii) a-type, perpendicular polarization:

$\frac{\mu_{a} E_{X}}{\sqrt{2}}(-1)^{M-P}\left[(2 J+1)\left(2 J^{\prime}+1\right)\right]^{1 / 2}\left[\left(\begin{array}{ccc}J & 1 & J^{\prime} \\ -M & -1 & M^{\prime}\end{array}\right)-\left(\begin{array}{ccc}J & 1 & J^{\prime} \\ -M & 1 & M^{\prime}\end{array}\right)\right]\left(\begin{array}{ccc}J & 1 & J^{\prime} \\ -P & 0 & P^{\prime}\end{array}\right) \delta_{\lambda, \lambda^{\prime}} \delta_{\sigma, \sigma^{\prime}} \delta_{\epsilon,-\epsilon^{\prime}}$

iii) $b$-type, parallel polarization:

$\frac{\mu_{b} E_{Z}}{2 \sqrt{2}}\left(1+\epsilon \epsilon^{\prime}\right)(-1)^{M-P}\left[(2 J+1)\left(2 J^{\prime}+1\right)\right]^{1 / 2}\left(\begin{array}{ccc}J & 1 & J^{\prime} \\ -M & 0 & M^{\prime}\end{array}\right) \times\left\{\left[\left(\begin{array}{ccc}J & 1 & J^{\prime} \\ -P & -1 & P^{\prime}\end{array}\right)-\left(\begin{array}{ccc}J & 1 & J^{\prime} \\ -P & 1 & P^{\prime}\end{array}\right)\right] \delta_{\lambda, \lambda^{\prime}} \delta_{\sigma, \sigma^{\prime}}-\right.$

$\left.\epsilon(-1)^{J-1 / 2}\left(\begin{array}{ccc}J & 1 & J^{\prime} \\ P & -1 & P^{\prime}\end{array}\right) \delta_{\lambda,-\lambda^{\prime}} \delta_{\sigma,-\sigma^{\prime}}\right\}$

iv) $b$-type, perpendicular polarization:

$$
\begin{aligned}
& \frac{\mu_{b} E_{X}}{4}\left(1+\epsilon \epsilon^{\prime}\right)(-1)^{M-P}\left[(2 J+1)\left(2 J^{\prime}+1\right)\right]^{1 / 2}\left[\left(\begin{array}{ccc}
J & 1 & J^{\prime} \\
-M & -1 & M^{\prime}
\end{array}\right)-\left(\begin{array}{ccc}
J & 1 & J^{\prime} \\
-M & 1 & M^{\prime}
\end{array}\right)\right] \times \\
& \left\{\left[\left(\begin{array}{ccc}
J & 1 & J^{\prime} \\
-P & -1 & P^{\prime}
\end{array}\right)-\left(\begin{array}{ccc}
J & 1 & J^{\prime} \\
-P & 1 & P^{\prime}
\end{array}\right)\right] \delta_{\lambda, \lambda^{\prime}} \delta_{\sigma, \sigma^{\prime}}-\epsilon(-1)^{J-1 / 2}\left(\begin{array}{ccc}
J & 1 & J^{\prime} \\
P & -1 & P^{\prime}
\end{array}\right) \delta_{\lambda,-\lambda^{\prime}} \delta_{\sigma,-\sigma^{\prime}}\right\}
\end{aligned}
$$

v) $c$-type, parallel polarization:

$$
\begin{aligned}
& \frac{-i \mu_{c} E_{Z}}{2 \sqrt{2}}\left(1-\epsilon \epsilon^{\prime}\right)(-1)^{M-P}\left[(2 J+1)\left(2 J^{\prime}+1\right)\right]^{1 / 2}\left(\begin{array}{ccc}
J & 1 & J^{\prime} \\
-M & 0 & M^{\prime}
\end{array}\right) \times\left\{\left[\left(\begin{array}{ccc}
J & 1 & J^{\prime} \\
-P & -1 & P^{\prime}
\end{array}\right)+\left(\begin{array}{ccc}
J & 1 & J^{\prime} \\
-P & 1 & P^{\prime}
\end{array}\right)\right] \delta_{\lambda, \lambda^{\prime}} \delta_{\sigma, \sigma^{\prime}}-\right. \\
& \left.\epsilon(-1)^{J-1 / 2}\left(\begin{array}{ccc}
J & 1 & J^{\prime} \\
P & -1 & P^{\prime}
\end{array}\right) \delta_{\lambda,-\lambda^{\prime}} \delta_{\sigma,-\sigma^{\prime}}\right\}
\end{aligned}
$$


vi) $c$-type, perpendicular polarization:

$$
\begin{aligned}
& \frac{\mu_{c} E_{Y}}{4}\left(1-\epsilon \epsilon^{\prime}\right)(-1)^{M-P}\left[(2 J+1)\left(2 J^{\prime}+1\right)\right]^{1 / 2}\left[\left(\begin{array}{ccc}
J & 1 & J^{\prime} \\
-M & -1 & M^{\prime}
\end{array}\right)+\left(\begin{array}{ccc}
J & 1 & J^{\prime} \\
-M & 1 & M^{\prime}
\end{array}\right)\right] \times \\
& \left\{\left[\left(\begin{array}{ccc}
J & 1 & J^{\prime} \\
-P & -1 & P^{\prime}
\end{array}\right)+\left(\begin{array}{ccc}
J & 1 & J^{\prime} \\
-P & 1 & P^{\prime}
\end{array}\right)\right] \delta_{\lambda, \lambda^{\prime}} \delta_{\sigma, \sigma^{\prime}}-\epsilon(-1)^{J-1 / 2}\left(\begin{array}{ccc}
J & 1 & J^{\prime} \\
P & -1 & P^{\prime}
\end{array}\right) \delta_{\lambda,-\lambda^{\prime}} \delta_{\sigma,-\sigma^{\prime}}\right\}
\end{aligned}
$$

Hybrid bands or random polarization configurations can be handled by an appropriate combination of eqns. 21 through 26.

\section{Stark Spectra Simulations}

Stark spectra are simulated in Figures 4 through 8 for both $a$ - and $b$-type rovibrational bands, restricted to either parallel $(\Delta M=0)$ or perpendicular polarization $(\Delta M= \pm 1)$ selection rules. Here we use the zero-field constants, line widths, and rotational temperature reported previously for He-solvated $\mathrm{OH}-\mathrm{C}_{2} \mathrm{H}_{2}$ (Table II) [23]. The value of the permanent electric dipole moment along the $a$-axis is set to 1.85 Debye.

The $a$-type, zero-field spectrum at the bottom of Figure 4 is labeled with quantum numbers associated with the weak-intermolecular interaction limit, ${ }^{\Delta P} \Delta J_{P^{\prime \prime}}\left(J^{\prime \prime}\right)$, although the simulations are carried out with $\rho=-165 \mathrm{~cm}^{-1}$. Transitions derived from parity doubled levels in the $\left(P=\frac{1}{2}, \omega=+\frac{3}{2}\right)$ manifold are shown beneath the spectrum, whereas, as discussed above, parity splitting is not resolved for transitions derived from levels in the $\left(P=\frac{3}{2}, \omega=+\frac{3}{2}\right)$ manifold. The asterisks located above the $2 \mathrm{kV} / \mathrm{cm}$ Stark simulation are meant to draw the eye to the pseudo-first-order Stark splitting associated with the levels in this latter manifold. 
The effect of a non-zero difference potential on the Stark splitting is shown in Figure 5 for the $a$-type band (perpendicular polarization). The field strength in these spectra is set to 4 $\mathrm{kV} / \mathrm{cm}$, and the value of $|\rho|$ increases from bottom to top in the figure. When $\rho=0$, each of the zero-field transitions (inverted red spectrum) is noticeably split by the Stark effect. As discussed above, this can be traced to the near degeneracy of the two opposite parity states at zero-field, which allows for a linear Stark effect. As the magnitude of the difference potential increases, however, parity doubling of levels in the $\left(P=\frac{1}{2}, \omega=+\frac{3}{2}\right)$ manifold acts to "de-tune" the interaction with the Stark field. The two states of opposite parity are mixed less as a result of this, and the Stark splitting of transitions derived from these levels is reduced.

Returning to simulations in which $\rho=-165 \mathrm{~cm}^{-1}$, as shown in Figure 6, the $a$-type, parallel polarization spectrum collapses into a single peak near the band origin at high field strengths $(>16 \mathrm{kV} / \mathrm{cm})$. This effect is the signature of the formation of pendular states characterized by the librational motion of the dipole about the field direction [28, 29]. This signature of the "brute-force orientation" of the dipole moment is equivalent to that previously observed for "parallel" bands of closed-shell polar molecules in the presence of large static electric fields [2].

The $b$-type spectrum at zero-field is shown at the bottom of Figures 7 and 8, along with the assignments of ${ }^{\Delta P} \Delta J_{P^{\prime \prime}}$ sub-bands. The line widths in these $b$-type simulations are about half the experimentally measured values [23], simply to show more detail. The spectrum is spread out over a much broader frequency range in comparison to the $a$-type spectrum, as a result of the nominal $\Delta P= \pm 1$ selection rules. As can be seen for the perpendicular polarization configuration (Figure 7), the spectrum becomes complicated under relatively low-field 
conditions due to the rapid Stark splitting of $P^{\prime \prime}=\frac{3}{2}$ levels. Similar to the $a$-type parallel polarization case, the spectrum becomes simplified in the high-field limit upon pendular state formation.

Experimental Stark spectra of $\mathrm{OH}-\mathrm{C}_{2} \mathrm{H}_{2}$ are compared to simulations for multiple field strengths in Figures 9, 10 and 11. Perpendicular polarization, $a$-type spectra in Figure 9 are best reproduced by simulations having slightly different vibrationally averaged dipole moments in the ground and excited (OH stretch) vibrational states. The dipole moments of the $T$-shaped $\mathrm{OH}-\mathrm{C}_{2} \mathrm{H}_{2}$ complex used in these simulations are $\mu^{\prime \prime}=1.85(2) \mathrm{D}$ and $\mu^{\prime}=1.90(2) \mathrm{D}$, where the uncertainty derives largely from the uncertainty in the field strength, which is calibrated by measurements of the Stark splitting of the $\mathrm{HCN} R(0)$ line under identical experimental conditions [30]. For comparison, the ground state dipole moment of He-solvated $\mathrm{OH}$ is 1.61(2) D [31]. The $a$-type parallel polarization (Figure 10) Stark spectra are also satisfactorily simulated with this set of dipole moments. The $b$-type spectra (CH antisymmetric stretch; Figure 11) do not require slightly different dipole moments in the simulations; although, because of the sixfold broader line widths, these spectra are less sensitive to small changes in dipole moment, in comparison to the $a$-type Stark spectra. Collectively, the rather remarkable agreement between experiment and simulation (keeping in mind that these are spectra of a weakly bound, Hesolvated molecular complex) confirms the suitability of the employed two-state model for systems of this type. We are now in a position to apply this model to the Stark spectra of a variety of $\mathrm{OH}$ containing molecular complexes, which are predicted as entrance channel complexes on the potential energy surfaces associated with reactions relevant to both atmospheric and combustion chemistry. 
Two additional sets of Stark spectra for similar systems are shown in Figures 12 and 13. The spectra in Figure 12 correspond to the $a$-type $\mathrm{OH}$ stretch band of the $T$-shaped $\mathrm{OH}-\mathrm{C}_{2} \mathrm{H}_{4}$ complex (Figure 1). Although these $a$-type spectra are broader than those associated with the $\mathrm{C}_{2} \mathrm{H}_{2}$ containing complex, an experimentally derived dipole moment of 2.10(4) D can be extracted from the spectra. Here the uncertainty is somewhat larger and limited to the sensitivity of the rotational contour to small changes in the dipole moment. We also note that the experimental zero-field spectrum isn't substantially different than a simulation assuming $\rho=0$. The simulations in Figure 12 assume $\rho=-25 \mathrm{~cm}^{-1}$, which leads to parity doubling sufficient to slightly broaden the ${ }^{q} R_{1 / 2}(1 / 2)$ and ${ }^{q} P_{1 / 2}(3 / 2)$ transitions in the zero-field spectrum. Zero-field $a$ type spectra of the OD- $\mathrm{C}_{2} \mathrm{H}_{4}$ have also been measured, and these are sharper, revealing a parity splitting of these aforementioned transitions consistent with a difference potential equal to -25 $\mathrm{cm}^{-1}$.

The spectra in Figure 13 correspond to the $a$-type $\mathrm{OH}$ stretch band of the $\mathrm{OH}-\mathrm{H}_{2} \mathrm{O}$ complex. Microwave spectra of these species reveal a vibrationally averaged, planar $C_{2 v}$ structure, where the $\mathrm{OH}$ moiety lies on the $a$-axis and hydrogen bonds to the oxygen atom of water (Figure 1) [24, 25]. The He droplet zero-field rovibrational spectrum of this species will be discussed in detail in a future report, which will also discuss the dipole moments of the binary complex and other $\mathrm{OH}-\left(\mathrm{H}_{2} \mathrm{O}\right)_{\mathrm{n}}$ clusters. Assuming a $C_{2 v}$ structure and $\rho=-146 \mathrm{~cm}^{-1}$, the twostate model was used to generate the simulations in Figure 13, from which dipole moments of $\mu^{\prime \prime}=3.70(5) \mathrm{D}$ and $\mu^{\prime}=3.75(5) \mathrm{D}$ can be extracted from the spectra. Table II is a collection of all constants and parameters used in the simulations of spectra in Figures 9-13. 


\section{Experimental Methods}

The helium droplet experiment, as applied to continuous wave, IR laser Stark spectroscopy of molecular complexes, has been discussed in detail elsewhere [32,33]. Briefly, He droplets with 4000 atoms on average are produced in a continuous, cryogenic nozzle expansion. The droplet expansion is skimmed into a beam and passes into a chamber containing the source of hydroxyl radicals. The $\mathrm{OH}$ radicals are produced via the pyrolytic decomposition of tert-butyl hydroperoxide, as described previously [10, 31, 34]. The helium droplets "pick-up" $\mathrm{OH}$ radicals as they emanate from this effusive pyrolysis source, and the internal degrees of freedom of the radical are cooled to less than $0.4 \mathrm{~K}$ via $\mathrm{He}$ atom evaporation [31]. Downstream from the $\mathrm{OH}$ pick-up zone, the $\mathrm{OH}$ doped droplet beam passes through a $2 \mathrm{~cm}$ long differentially pumped gas cell that contains $\approx 10^{-6}$ Torr of either $\mathrm{C}_{2} \mathrm{H}_{2}, \mathrm{C}_{2} \mathrm{H}_{4}$, or $\mathrm{H}_{2} \mathrm{O}$. Approximately 30 percent of the $\mathrm{OH}$ doped droplets pick-up one closed-shell molecule, which is also cooled to below $0.4 \mathrm{~K}$ [35], and within about $1 \mathrm{~ns}$, the $\mathrm{OH}$ and closed-shell fragments find each other to form a molecular complex.

The doped droplets then pass through the center of a Stark multi-pass cell, which consists of two parallel, gold-coated mirrors and two orthogonally placed, stainless steel electrodes. The electrodes are parallel and separated by about $3 \mathrm{~mm}$. Approximately 50 laser passes intersect the droplet beam as it travels through the homogeneous dc electric field. The field strength is calibrated via the Stark splitting of the $\operatorname{HCN} R(0)$ line [30]. Vibrational excitation of the dopant leads to the evaporation of several hundred helium atoms, which is detected as a depletion in the 
electron impact ionization cross-section of the droplet beam, using a quadrupole mass spectrometer. The IR laser is mechanically chopped, and the ion signal is processed with a lockin amplifier as the laser is tuned with $\approx 10 \mathrm{MHz}$ resolution [36]. A high-precision wavemeter is used as the frequency axis (absolute and relative frequency calibration), and the ion depletion signal is normalized to laser power.

\section{Summary}

Rovibrational Stark spectra of several hydroxyl radical containing complexes have been measured in helium droplets. A model is presented here that can account for the Stark effect in these systems, which all exhibit partial quenching of electronic angular momentum quantized along the $\mathrm{OH}$ bond axis. The spherical tensor operator formalism is employed so as to arrive at the most general solution, in which the permanent dipole moment has projections on all three inertial axes; however, the situation specifically addressed here is for $C_{2 v}$ complexes, in which the $\mathrm{OH}$ bond axis defines both the $a$-inertial axis and the direction of the permanent dipole moment. Formula for transition intensities are given for $a-, b$-, and $c$-type bands measured with either parallel or perpendicular laser polarization configurations.

For these open-shell complexes, a Coriolis interaction of the form $\hat{J}_{a}\left(\hat{l}_{a}+\hat{s}_{a}\right)$ lifts the $\pm \omega$ degeneracy associated with the electronic angular momentum of the $\mathrm{OH}$ radical. This dramatically reduces the effect of the inertial asymmetry in these complexes, and in the absence of angular momentum quenching, all rotational levels exhibit a pseudo-first-order Stark effect. In the presence of angular momentum quenching, a subset of levels is parity doubled (levels in the $\left(P=\frac{1}{2}, \omega=+\frac{3}{2}\right)$ manifold), removing the degeneracy responsible for the linear Stark effect. 
Stark splitting of these parity doubled levels is consistent with the quadratic Stark effect expected for non-degenerate levels of a closed-shell asymmetric top.

For the $T$-shaped $\mathrm{OH}-\mathrm{C}_{2} \mathrm{H}_{2}$ complex, predictions of $a$ - and $b$-type bands for a variety of Stark field strengths are generally in excellent agreement with experiment. The uncertainty in the dipole moments extracted from these spectra is limited by the uncertainty in the electric field strength. The model produces simulations that are also in excellent agreement with two other systems, $\mathrm{OH}-\mathrm{C}_{2} \mathrm{H}_{2}$ and $\mathrm{OH}-\mathrm{H}_{2} \mathrm{O}$, although the uncertainty in the dipole moments is somewhat larger due to broader line widths in the experimental helium droplet spectra of these species.

\section{Acknowledgements}

We acknowledge support from the Office of Energy Research, Office of Basic Energy Sciences, Chemical Sciences, Geosciences and Biosciences Division of the US Department of Energy (DOE) under Contract No. DE-FG02-12ER16298.

\section{References}

[1] J. Brown, A. Carrington, Rotational Spectroscopy of Diatomic Molecules, Cambridge University Press, Cambridge, 2003.

[2] D.T. Moore, L. Oudejans, R.E. Miller, Pendular state spectroscopy of an asymmetric top: Parallel and perpendicular bands of acetylene-HF, J. Chem. Phys., 110 (1999) 197-208.

[3] T.C. Steimle, Permanent electric dipole moments of metal containing molecules, Int. Rev. Phys. Chem., 19 (2000) 455-477. 
[4] P.R. Bunker, P. Jensen, Molecular Symmetry and Spectroscopy, NRC Research Press, Ottawa, 1998.

[5] D. Ajitha, S. Pal, Dipole moments of open-shell radicals using an analytic linear response approach in the Fock space multi-reference coupled cluster method, Chem. Phys. Lett., 309 (1999) 457-462.

[6] P.U. Manohar, S. Pal, Dipole moments and polarizabilities of some small radicals using constrained variational response to Fock-space multi-reference coupled-cluster theory, Chem. Phys. Lett., 438 (2007) 321-325.

[7] L. Ravichandran, D. Bhattacharya, N. Vaval, S. Pal, Fock-space multi-reference coupledcluster response with the effect of triples on dipole moment of $\mathrm{ClO}$ and SF radicals, J Chem Sci, 124 (2012) 223-232.

[8] F. Jensen, Polarization consistent basis sets: Principles, J. Chem. Phys., 115 (2001) 91139125.

[9] F. Jensen, Polarization consistent basis sets. III. The importance of diffuse functions, J. Chem. Phys., 117 (2002) 9234-9240.

[10] T. Liang, D.B. Magers, P.L. Raston, W.D. Allen, G.E. Douberly, Dipole Moment of the HOOO Radical: Resolution of a Structural Enigma, J. Phys. Chem. Lett., 4 (2013) 3584-3589.

[11] M. Schnell, P.R. Bunker, G. von Helden, J.U. Grabow, G. Meijer, A. van der Avoird, Stark Effect in the Benzene Dimer, Journal of Physical Chemistry A, 117 (2013) 13775-13778.

[12] M. Lemeshko, R.V. Krems, J.M. Doyle, S. Kais, Manipulation of molecules with electromagnetic fields, Mol. Phys., 111 (2013) 1648-1682.

[13] J.J. Gilijamse, S. Hoekstra, S.Y.T. van de Meerakker, G.C. Groenenboom, G. Meijer, Nearthreshold inelastic collisions using molecular beams with a tunable velocity, Science, 313 (2006) 1617-1620.

[14] M. Kirste, X.A. Wang, H.C. Schewe, G. Meijer, K.P. Liu, A. van der Avoird, L.M.C. Janssen, K.B. Gubbels, G.C. Groenenboom, S.Y.T. van de Meerakker, Quantum-State Resolved 
Bimolecular Collisions of Velocity-Controlled OH with NO Radicals, Science, 338 (2012) 10601063.

[15] M. Brouard, H. Chadwick, C.J. Eyles, B. Hornung, B. Nichols, J.M. Scott, F.J. Aoiz, J. Klos, S. Stolte, X. Zhang, The fully quantum state-resolved inelastic scattering of $\mathrm{NO}(\mathrm{X})$ plus Ne: experiment and theory, Mol. Phys., 111 (2013) 1759-1771.

[16] I.C. Bowater, J.M. Brown, Carringt.A, Microwave Spectroscopy of Nonlinear Free-Radicals 1. General Theory and Application to Zeeman Effect in HCO, P. Roy. Soc. Lond. A, Mat. Phys. Sci., 333 (1973) 265-288.

[17] M. Garttner, J.J. Omiste, P. Schmelcher, R. Gonzalez-Ferez, Fine structure of open-shell diatomic molecules in combined electric and magnetic fields, Mol. Phys., 111 (2013) 1865-1878.

[18] P.D.A. Mills, C.M. Western, B.J. Howard, Rotational Spectra of Rare-Gas Nitric-Oxide Van der waals Molecules 1. Theory of the Rotational Energy-Levels, J. Phys. Chem., 90 (1986) 33313338.

[19] J.M. Merritt, J. Küpper, R.E. Miller, Entrance channel X-HF (X = Cl, Br and I) complexes studied by high-resolution infrared laser spectroscopy in helium nanodroplets, Phys. Chem. Chem. Phys., 7 (2005) 67-78.

[20] M.D. Marshall, M.I. Lester, Spectroscopic implications of the coupling of unquenched angular momentum to rotation in OH-containing complexes, J. Chem. Phys., 121 (2004) 30193029.

[21] M.D. Marshall, M.I. Lester, Spectroscopic implications of partially quenched orbital angular momentum in the OH-water complex, J. Phys. Chem. B, 109 (2005) 8400-8406.

[22] M.D. Marshall, J.B. Davey, M.E. Greenslade, M.I. Lester, Evidence for partial quenching of orbital angular momentum upon complex formation in the infrared spectrum of $\mathrm{OH}$-acetylene, $\mathrm{J}$. Chem. Phys., 121 (2004) 5845-5851.

[23] G.E. Douberly, P.L. Raston, T. Liang, M.D. Marshall, Infrared Rovibrational Spectroscopy of $\mathrm{OH}-\mathrm{C}_{2} \mathrm{H}_{2}$ in ${ }^{4} \mathrm{He}$ Nanodroplets: Paritty Splitting due to Partially Quenched Electronic Angular Momentum, J. Chem. Phys., 142 (2015) 134306. 
[24] C.S. Brauer, G. Sedo, E.M. Grumstrup, K.R. Leopold, M.D. Marshall, H.O. Leung, Effects of partially quenched orbital angular momentum on the microwave spectrum and magnetic hyperfine splitting in the OH-water complex, Chem. Phys. Lett., 401 (2005) 420-425.

[25] C.S. Brauer, G. Sedo, E. Dahlke, S.H. Wu, E.M. Grumstrup, K.R. Leopold, M.D. Marshall, H.O. Leung, D.G. Truhlar, Effects of (18)O isotopic substitution on the rotational spectra and potential splitting in the $\mathrm{OH}-\mathrm{OH}(2)$ complex: Improved measurements for (16)OH-(16)OH(2) and (18) $\mathrm{OH}-(18) \mathrm{OH}(2)$, new measurements for the mixed isotopic forms, and ab initio calculations of the (2)A '-(2)A " energy separation, J. Chem. Phys., 129 (2008) 104304.

[26] S.H. Wu, G. Sedo, K.R. Leopold, Microwave spectrum of the $\mathrm{OD}-\mathrm{OH}_{2}$ complex: A strong deuterium isotope effect on angular momentum quenching in the hydroxyl moiety, J. Mol. Spec., 253 (2009) 35-40.

[27] W.M. Fawzy, J.T. Hougen, Rotational Energy-Levels and Line-Intensities for 2s+1Lambda-2s+1-Lambda and 2s+1(Lambda+/-1)-2s+1-Lambda Transitions in a Diatomic Molecule Vanderwaals Bonded to a Closed Shell Partner, J. Mol. Spec., 137 (1989) 154-165. [28] B. Friedrich, D.R. Herschbach, Spatial Orientation of Molecules in Strong Electric-Fields and Evidence for Pendular States, Nature, 353 (1991) 412-414.

[29] J.M. Rost, J.C. Griffin, B. Friedrich, D.R. Herschbach, Pendular States and Spectra of Oriented Linear-Molecules, Phys. Rev. Lett., 68 (1992) 1299-1301.

[30] P.L. Stiles, K. Nauta, R.E. Miller, Dipole moments of molecules solvated in helium nanodroplets, Phys. Rev. Lett., 90 (2003) 135301.

[31] P.L. Raston, T. Liang, G.E. Douberly, Anomolous Lambda-Doubling in the Infrared Spectrum of the Hydroxyl Radical in Helium Nanodroplets, Journal of Physical Chemistry A, 117 (2013) 8103-8110.

[32] J.P. Toennies, A.F. Vilesov, Superfluid helium droplets: A uniquely cold nanomatrix for molecules and molecular complexes, Angew. Chem. Int. Edit., 43 (2004) 2622-2648.

[33] M.Y. Choi, G.E. Douberly, T.M. Falconer, W.K. Lewis, C.M. Lindsay, J.M. Merritt, P.L. Stiles, R.E. Miller, Infrared spectroscopy of helium nanodroplets: novel methods for physics and chemistry, Int. Rev. Phys. Chem., 25 (2006) 15-75. 
[34] P.L. Raston, T. Liang, G.E. Douberly, Infrared spectroscopy of HOOO and DOOO in ${ }^{4} \mathrm{He}$ nanodroplets, J. Chem. Phys., 137 (2012) 184302.

[35] M. Hartmann, R.E. Miller, J.P. Toennies, A. Vilesov, Rotationally Resolved Spectroscopy of $\mathrm{SF}_{6}$ in Liquid-Helium Clusters - A Molecular Probe of Cluster Temperature, Phys. Rev. Lett., 75 (1995) 1566-1569.

[36] A.M. Morrison, T. Liang, G.E. Douberly, Automation of an "Aculight" continuous-wave Optical Parametric Oscillator, Review of Scientific Instruments, 84 (2013) 013102. 
TABLE I. Transformation properties of parity conserving basis functions.

\begin{tabular}{|c|c|c|}
\hline & \multicolumn{2}{|c|}{$(P+\sigma)$} \\
\hline & Even & Odd \\
\hline$\epsilon=+1$ & $A_{1}$ & $B_{1}$ \\
\hline$\epsilon=-1$ & $A_{2}$ & $B_{2}$ \\
\hline
\end{tabular}


TABLE II. Spectroscopic parameters (in $\mathrm{cm}^{-1}$ ) determined from simulations of the $a$ - and $b$-type spectra of $\mathrm{OH}-\mathrm{C}_{2} \mathrm{H}_{2}$ and $a$-type spectra of $\mathrm{OH}-\mathrm{C}_{2} \mathrm{H}_{4}$ and $\mathrm{OH}-\mathrm{H}_{2} \mathrm{O}$. ${ }^{\mathrm{a}}$

\begin{tabular}{|l|l|l|l|l|}
\hline & $\mathrm{OH}-\mathrm{C}_{2} \mathrm{H}_{2}(a$-type $)$ & $\mathrm{OH}-\mathrm{C}_{2} \mathrm{H}_{2}(b$-type $)$ & $\mathrm{OH}-\mathrm{C}_{2} \mathrm{H}_{4}(a$-type $)$ & $\mathrm{OH}-\mathrm{H}_{2} \mathrm{O}(a$-type $)$ \\
\hline$\nu_{0}$ & $3525.265(1)$ & $3278.800(1)$ & $3521.711(2)$ & $3485.815(3)$ \\
\hline$\mu^{\prime \prime}$ & $1.85(2)$ & $1.85(4)$ & $2.10(4)$ & $3.70(5)$ \\
\hline$\mu^{\prime}$ & $1.90(2)$ & $1.85(4)$ & $2.15(4)$ & $3.75(5)$ \\
\hline$A^{\prime \prime}$ & 1.186 & 1.186 & 0.41 & 13.4 \\
\hline$\Delta A^{\mathrm{b}}$ & -0.008 & -0.254 & 0 & -0.29 \\
\hline$(B+C) / 2^{\prime \prime}$ & 0.0605 & 0.0605 & 0.0525 & 0.09 \\
\hline$\Delta(B+C) / 2^{\mathrm{b}}$ & -0.0007 & -0.0007 & 0 & 0 \\
\hline$(B-C) / 2^{\prime \prime}$ & 0.0001 & 0.0001 & 0.0015 & 0 \\
\hline$\Delta(B-C) / 2^{\mathrm{b}}$ & 0 & 0 & 0 & 0 \\
\hline$D_{J}{ }^{\mathrm{c}}$ & 0.00029 & 0.00029 & 0.00029 & 0.00046 \\
\hline$D_{K}{ }^{\mathrm{c}}$ & 0.0078 & 0.0078 & 0.0078 & 0.0078 \\
\hline$\rho^{\mathrm{c}}$ & -165 & -165 & -25 & -146 \\
\hline$\Gamma$ & 0.008 & 0.05 & 0.027 & 0.08 \\
\hline$T_{r o t}(\mathrm{~K})$ & 0.35 & 0.35 & 0.35 & 0.35 \\
\hline
\end{tabular}

${ }^{a}$ Numbers in parentheses are $2 \sigma$ uncertainties in the last digit. The spin-orbit coupling constant was fixed at $-139.0508 \mathrm{~cm}^{-1}$ in the simulations.

${ }^{\mathrm{b}}$ The change is taken as the excited state minus the ground state constant.

${ }^{\mathrm{c}}$ The ground and excited state constants are the same. 


\section{FIGURE CAPTIONS}

Figure 1: Structures and coordinate system used in the analysis of the Stark spectra of $\mathrm{OH}-\mathrm{C}_{2} \mathrm{H}_{2}, \mathrm{OH}-\mathrm{C}_{2} \mathrm{H}_{4}$, and $\mathrm{OH}-\mathrm{H}_{2} \mathrm{O}$ complexes.

Figure 2: Simulated zero-field, $a$-type, $\mathrm{OH}$ stretch spectra for the $\mathrm{OH}-\mathrm{C}_{2} \mathrm{H}_{2}$ complex. The value of the difference potential is given above each spectrum. Rotational constants, line widths and rotational temperature are those determined previously from experiment [23]. The red spectrum most closely resembles the experimental spectrum of the He-solvated complex. The small splitting observed in the $\rho=0$ spectrum is due to a change in the $A$ rotational constant equal to $0.01 \mathrm{~cm}^{-1}$, rather than a residual parity splitting in the absence of the difference potential.

Figure 3: Stark splitting of the $\left|J=\frac{1}{2}, P=\frac{1}{2}, \omega=\frac{3}{2}\right\rangle$ (red), $\left|J=\frac{3}{2}, P=\frac{1}{2}, \omega=\frac{3}{2}\right\rangle$ (blue) and $\left|J=\frac{3}{2}, P=\frac{3}{2}, \omega=\frac{3}{2}\right\rangle$ (black) levels. The red and black levels are the lowest energy of $A$ and $B$ nuclear spin symmetry, respectively. The quantum numbers used to label the states are only valid in the zero-field, weak-intermolecular interaction limit, although $M$ remains a good quantum number throughout.

Figure 4: Simulations of Stark spectra constrained to $a$-type and perpendicular laser polarization selection rules. Parity splitting of ${ }^{q} \Delta J_{1 / 2}$ transitions in the zero-field spectrum is shown along the bottom of the figure $\left(\rho=-165 \mathrm{~cm}^{-1}\right)$. The asterisks are to guide the eye towards the pseudo first-order Stark splitting of the ${ }^{q} \Delta J_{3 / 2}$ transitions, which are derived from the non-parity doubled levels in the $\left|P=\frac{3}{2}, \omega=\frac{3}{2}\right\rangle$ manifold. 
Figure 5: The $4 \mathrm{kV} / \mathrm{cm}$ Stark spectrum (a-type, perpendicular polarization) simulated for different values of the difference potential. The zero-field spectrum with $\rho=0$ is shown inverted (red) along the bottom of the spectrum.

Figure 6: Simulations of Stark spectra constrained to $a$-type and parallel laser polarization selection rules $\left(\rho=-165 \mathrm{~cm}^{-1}\right)$.

Figure 7: Simulations of Stark spectra constrained to $b$-type and perpendicular laser polarization selection rules $\left(\rho=-165 \mathrm{~cm}^{-1}\right)$. The assignments of ${ }^{\Delta P} \Delta J_{P^{\prime \prime}}$ sub-bands are shown above the zero-field spectrum.

Figure 8: Simulations of Stark spectra constrained to $b$-type and parallel laser polarization selection rules $\left(\rho=-165 \mathrm{~cm}^{-1}\right)$. The assignments of ${ }^{\Delta P} \Delta J_{P^{\prime \prime}}$ sub-bands are shown above the zero-field spectrum.

Figure 9: Experimental and simulated Stark spectra of the $a$-type $\mathrm{OH}$ stretch band of the $\mathrm{OH}-\mathrm{C}_{2} \mathrm{H}_{2}$ complex. The laser polarization is aligned perpendicular to the Stark field axis.

Figure 10: Experimental and simulated Stark spectra of the $a$-type $\mathrm{OH}$ stretch band of the $\mathrm{OH}-\mathrm{C}_{2} \mathrm{H}_{2}$ complex. The laser polarization is aligned parallel to the Stark field axis.

Figure 11: Experimental and simulated Stark spectra of the $b$-type $\mathrm{CH}$ antisymmetric stretch band of the $\mathrm{OH}-\mathrm{C}_{2} \mathrm{H}_{2}$ complex. The laser polarization alignment is either parallel or perpendicular to the Stark field for the spectra on the left-hand and right-hand side of the figure, respectively. 
Figure 12: Experimental and simulated zero-field and Stark spectra of the $a$-type OH stretch band of the $\mathrm{OH}-\mathrm{C}_{2} \mathrm{H}_{4}$ complex. The laser polarization is aligned perpendicular to the Stark field axis.

Figure 13: Experimental and simulated Stark spectra of the $a$-type $\mathrm{OH}$ stretch band of the $\mathrm{OH}-\mathrm{H}_{2} \mathrm{O}$ complex. The laser polarization alignment is either perpendicular or parallel to the Stark field for the spectra on the left-hand and right-hand side of the figure, respectively. 


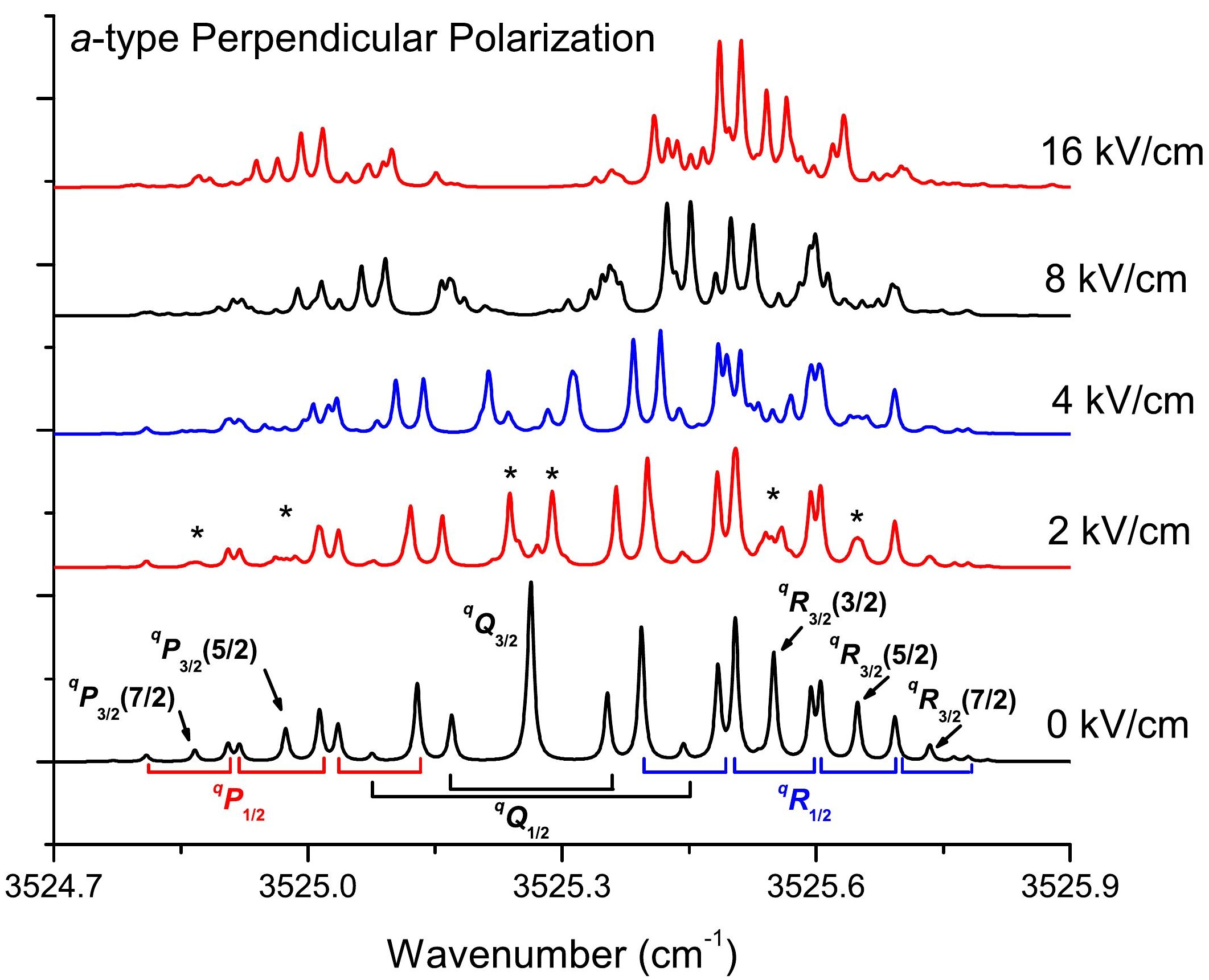


] a-type Parallel Polarization

$16 \mathrm{kV} / \mathrm{cm}$

$8 \mathrm{kV} / \mathrm{cm}$
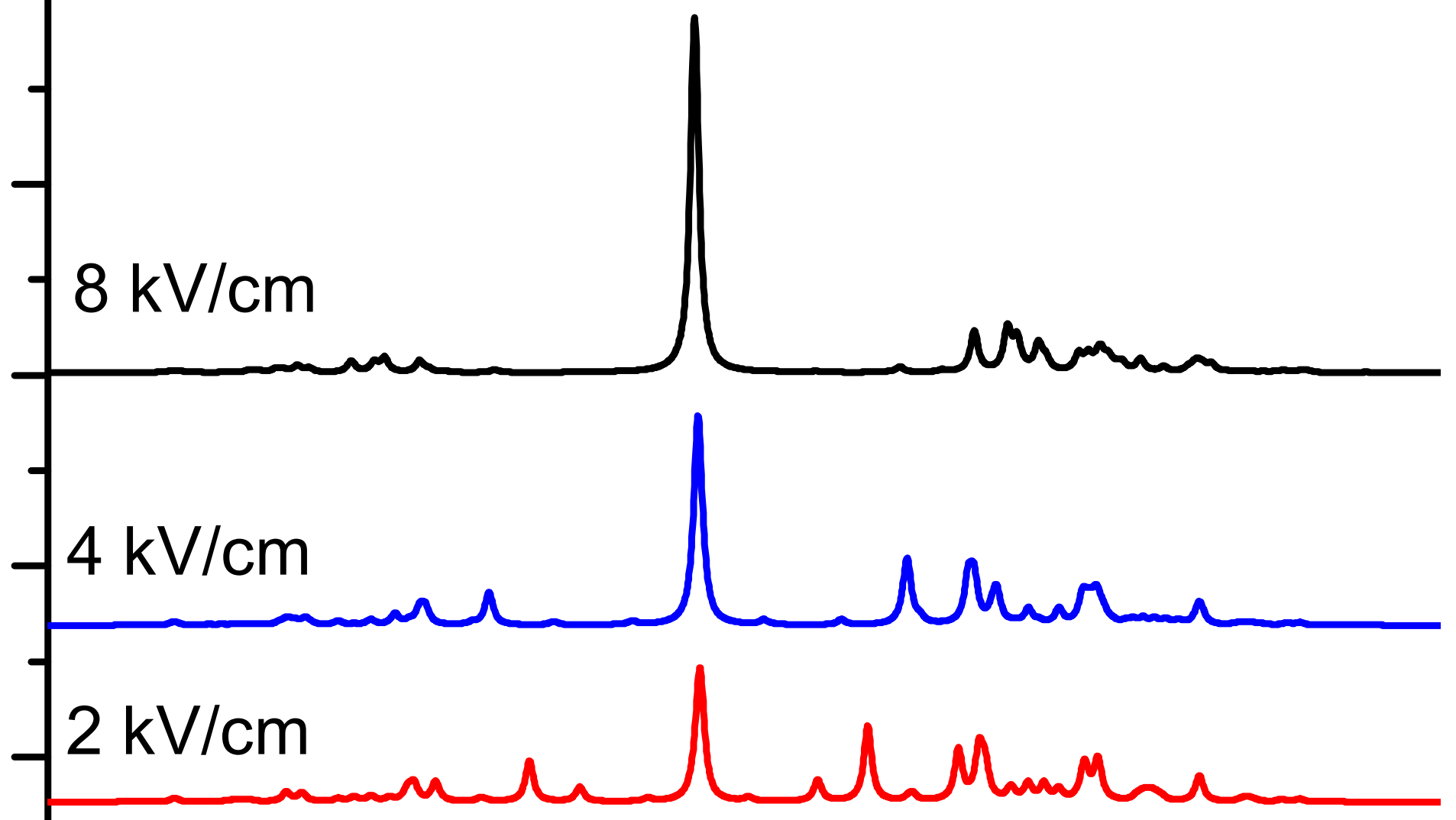

$-0 \mathrm{kV} / \mathrm{cm}$

\begin{tabular}{lllll}
\hline 3524.7 & 3525.0 & 3525.3 & 3525.6 & 3525.9
\end{tabular}

Wavenumber $\left(\mathrm{cm}^{-1}\right)$ 


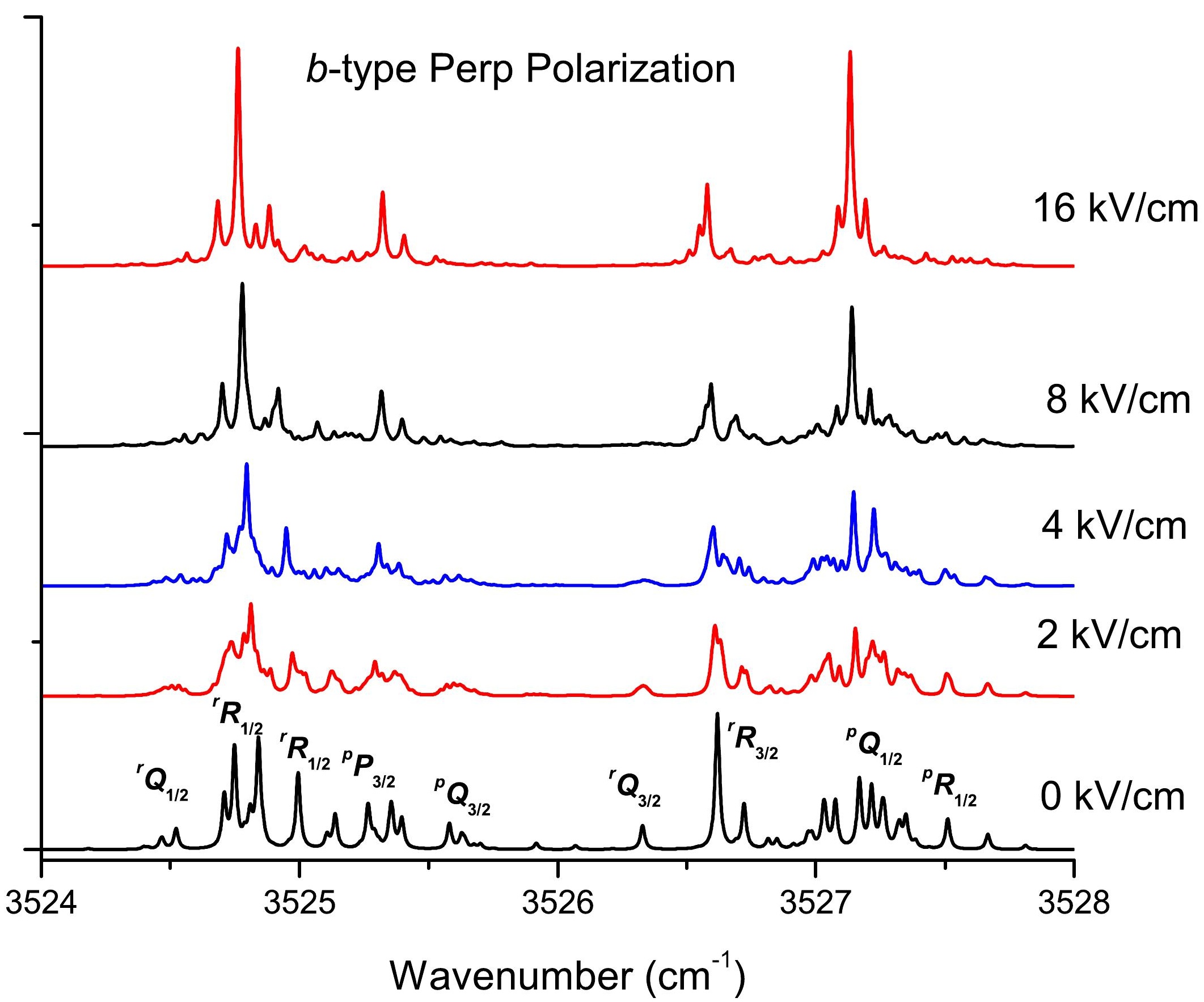




$$
\begin{aligned}
& \mu^{\prime \prime}=1.85(2) D \\
& \mu^{\prime}=1.90(2) D
\end{aligned}
$$
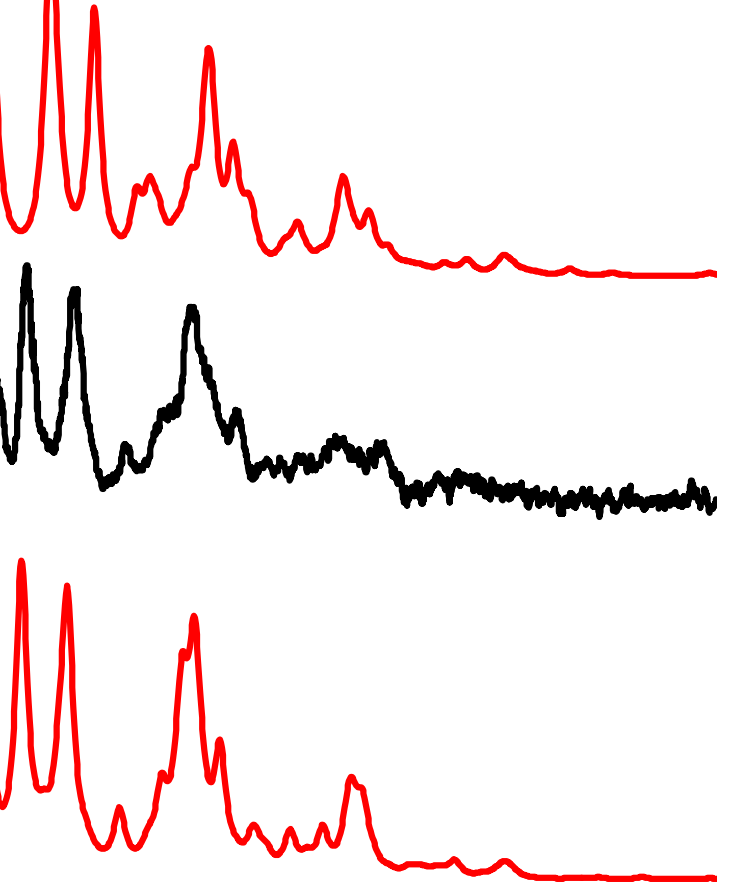
$3.56 \mathrm{kV/cm}$

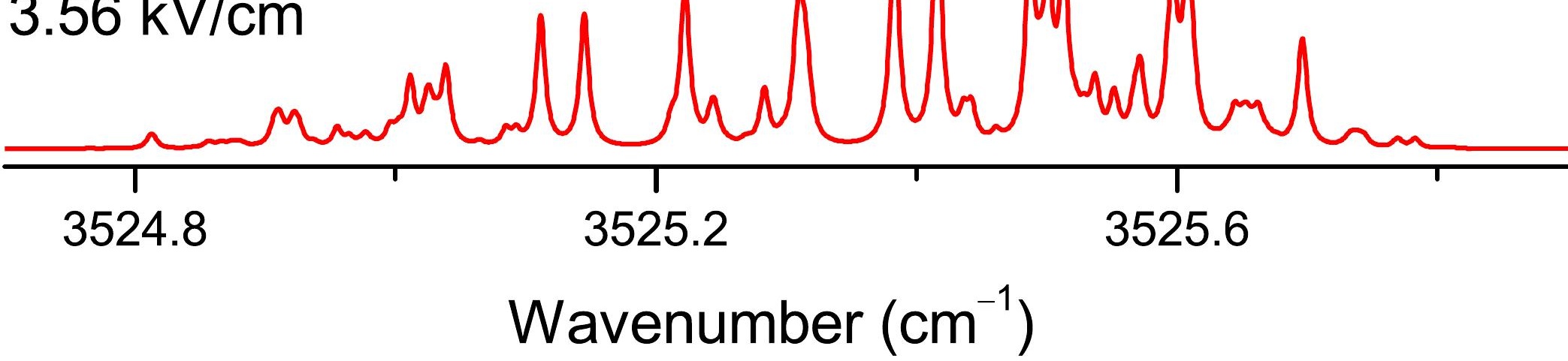




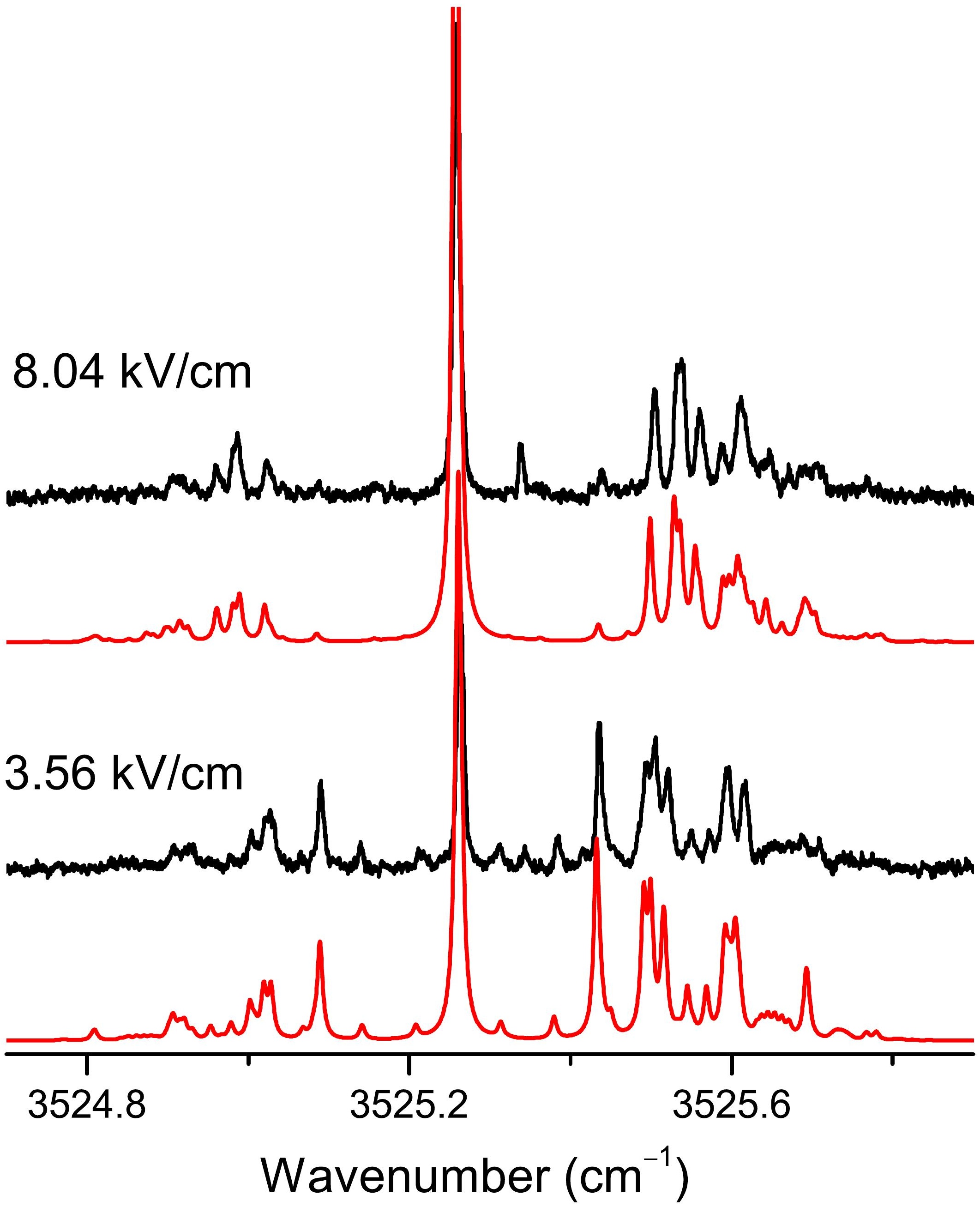

Figure

$8.04 \mathrm{kV} / \mathrm{cm}$

$3.56 \mathrm{kV} / \mathrm{cm}$

$$
\begin{array}{lc}
3524.8 & 3525.2 \\
& \text { Wavenumber }\left(\mathrm{cm}^{-1}\right)
\end{array}
$$


Perp Polarization

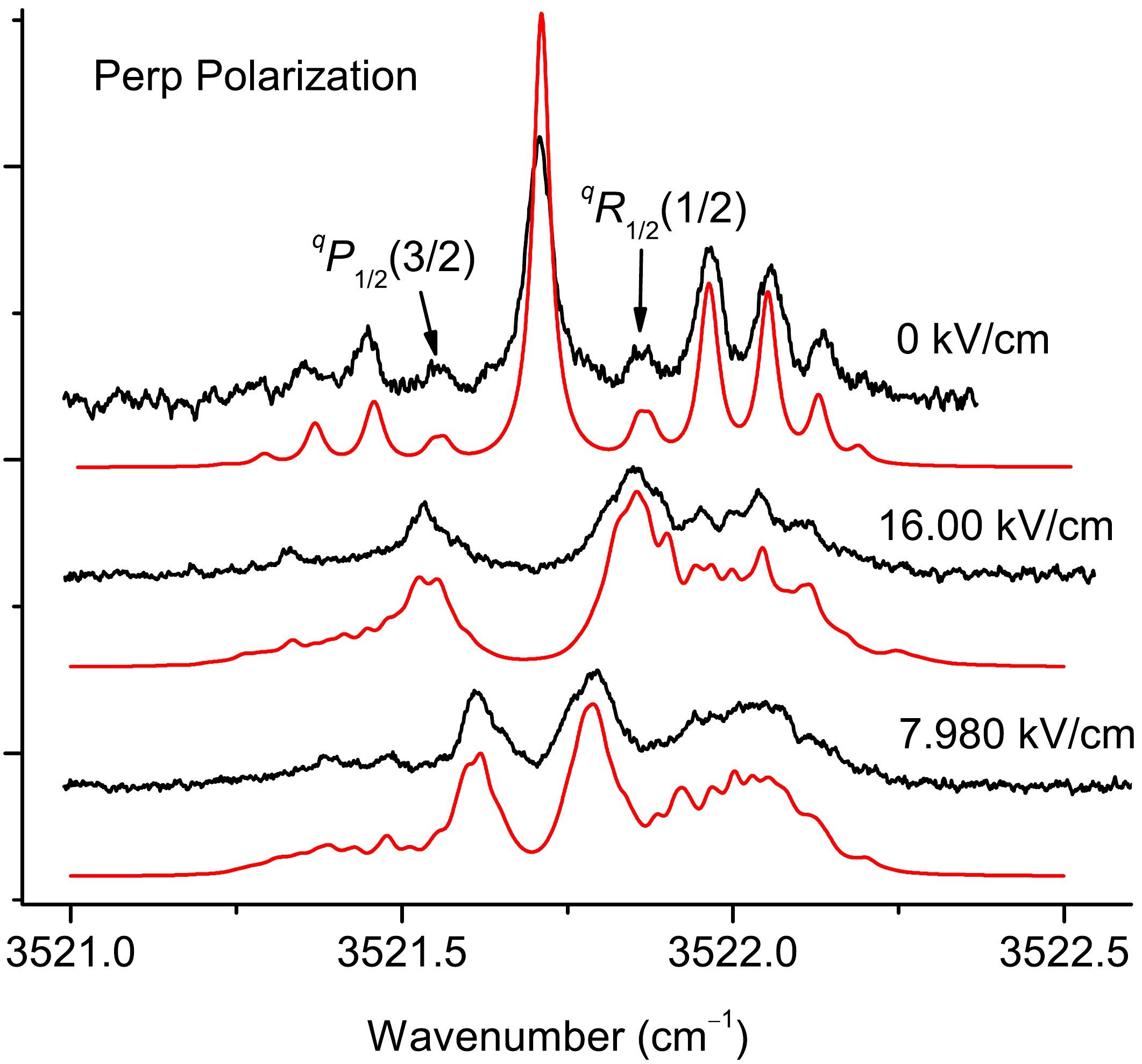




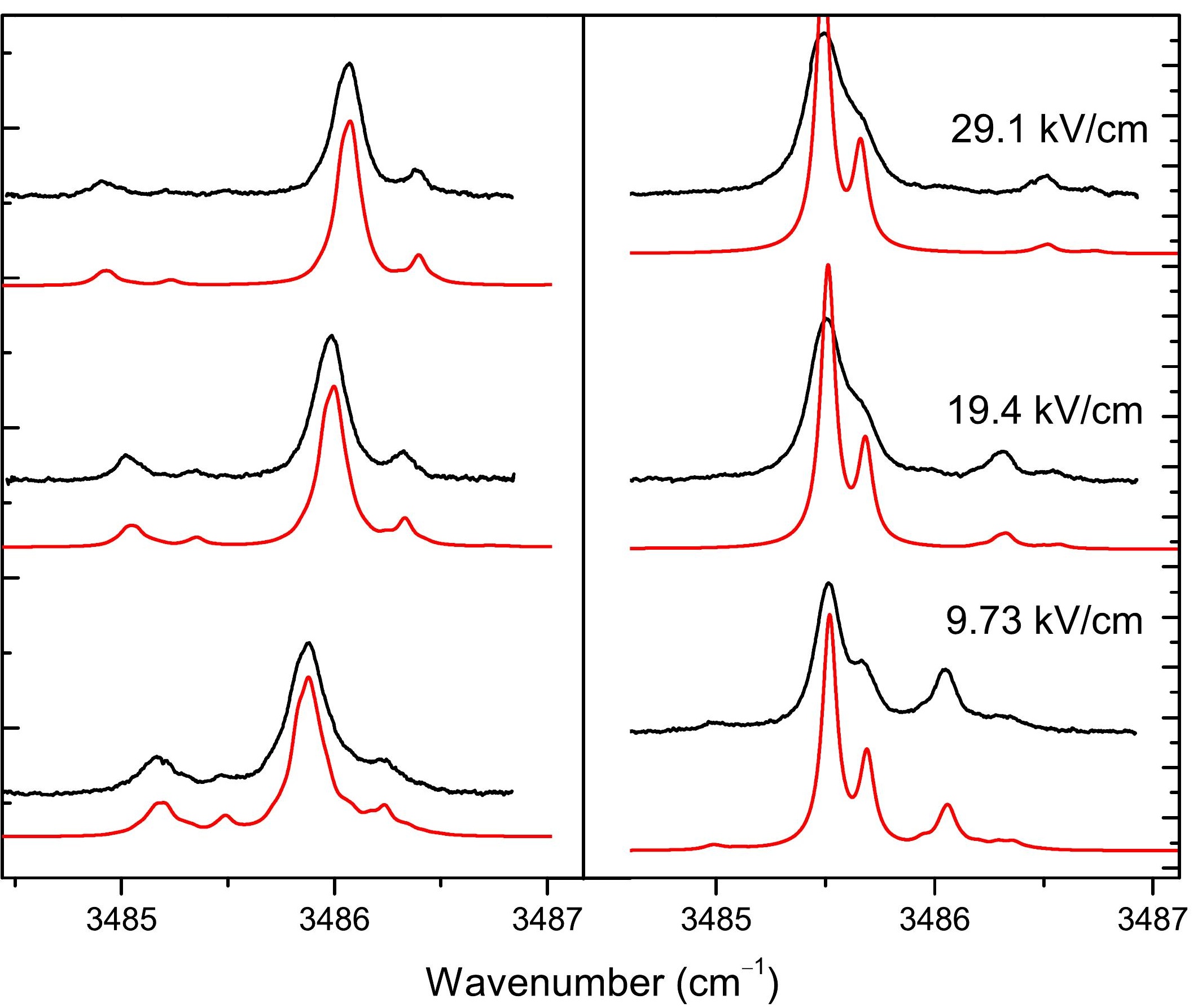

